\title{
LINEAR TIME-VARYING FEEDBACK LAW FOR VEHICLES WITH ACKERMANN STEERING
}

\begin{abstract}
In this paper, we propose an optimal state feedback control law for addressing point stabilization and tracking problems of nonholonomic vehicles with Ackermann steering in a unified manner. Unlike other feedback controllers that perform dynamic linearization of vehicle models, the proposed optimal feedback controller provides the state feedback control to the original nonlinear vehicle model for achieving excellent state-tracking performance. In addition, nonlinear control techniques suggested in the literature to date require that the desired trajectory of the robot is generated using persistently excited inputs. This may be too restrictive and non-realistic hypothesis to mimic a real scenario. Here, we address this issue by developing a smooth state-feedback control law that is formulated by modifying the classical Pontryagin's minimum principle. The proposed control law can be applied for solving control problems of a general class of nonlinear affine systems. The proposed control scheme offers a modular solution to other control techniques for a large number of mobile robot applications. The theoretical results are validated through computer simulations.
\end{abstract}

\section{Key Words}

Ackermann Steering, Hamiltonian, Mobile Robots, Optimal Feedback Gain 


\section{Introduction}

Global asymptotic solutions of point stabilization and tracking problems of nonlinear affine systems are still among the principle interests to the control community. A nonholonomic vehicle with Ackermann steering is a nonlinear affine system where the Brockett's theorem proves the nonexistence of smooth state-feedbacks for its asymptotic stabilization on fixed configurations as pointed out in [1]. Here, we propose a promising alternative solution to the Brockett's problem for a nonholonomic car-like vehicle (Ackermann steering vehicle) with a linear time-varying state-feedback law. The proposed optimal feedback law is determined by modifying the classical Pontryagin's minimum principle [2]. By doing so, both point stabilization and trajectory tracking problems of a nonholonomic vehicle are addressed as a unified manner. In addition, the motivation of the proposed linear time-varying feedback law stems from the fact that most of the research work suggested in the literature are tailored towards developing complex nonlinear control laws to address tracking problems of a simple unicycle-like robots. These control laws are powerful but they generally may be quite complex. As such, we emphasize that there exists a linear time-varying control law to address the tracking problem of nonlinear affine systems (in this paper, we consider vehicle with Ackermann steering), where the control law is easy to understand in the sense that all what a vehicle needs to track a pre-defined trajectory is a linear feedback operator which will be detailed in section 3. Note that the proposed control law can easily be coupled with navigation techniques for solving the robot navigation problem [3, 4].

Nonlinear feedback laws enable nonholonomic mobile robots to track a pre-defined trajectory, to stabilize on fixed configurations, or to synchronize among multiple robots [5, 6]. These laws have been explored through a variety of control techniques, such as differen-

tial flatness and back-stepping [7, 8, 9], nonlinear control coupled with data fusion algorithms [10, 11], and sliding mode control [12, 13, 14]. Recently, the trajectory tracking and 
the set-point stabilization problems of unicycle-type vehicles have been addressed in [15]. A few papers have addressed the trajectory tracking problem of nonlinear affine systems using time-varying feedback laws coupled with heuristics (see [16, 17, 18]). These techniques are quite powerful to solve trajectory tracking problems of nonlinear affine systems but require complex feedback law even for a simple unicycle-like affine systems [19]. In some cases, the satisfactory tracking performance is achieved at the cost of vehicle's model simplification, see [20, 21], for example. Model predictive control techniques are quite popular and have been extensively used for solving tracking problems of mobile robots in the optimal control literature, see [22, 23, 24], however, they suffer from defining appropriate feedback laws for partially observed states. Authors in [25, 26, 27] have recently adopted model predictive control laws for solving the tracking problems of nonholonomic systems. Their results are satisfactory at the cost of hardware needed to implement the control laws. Some researchers tackled tracking and stabilization problems separately. See [28, 29, 30, 31], for tracking problems, and [32, 33, 34] for stabilization problems. The trajectory tracking problem for nonholonomic vehicles has been tackled by transverse function approach [35]. A salient feature of this approach is the obtention of feedback laws that unconditionally achieve the practical stabilization of arbitrary reference trajectories, including fixed points and nonadmissible trajectories. However, this approach requires comprehensive tuning of transverse function parameters.

As a footnote, the aforementioned control techniques yield satisfactory tracking or stabilization performance. They either require the model simplification by tuning its parameters or need extensive derivations for feedback law even to solve problems of a simple unicycle-like vehicle. To overcome some of these issues, such as the model simplification, for example, Miah et al in [36, 37] introduced both time-varying and time-invariant feedback operators for solving tracking problems of a class of semi-linear and affine nonlinear dynamic systems using Pontryagin's minimum principle. These ideas are then exploited in solving tracking and 
regulation problems of differential drive mobile robots in indoor environments (see [38, 39], for example). This paper advances previous theoretical ideas using vehicles with Ackermann geometry in tracking a pre-defined trajectories or in parking on a fixed configuration in finite time (pre-defined). The work presented in [38] deals with an output (measurement) feedback control law as opposed to state-feedback control law presented in this manuscript. In [39], a state-feedback control law coupled with estimation is presented. As can be noted, both papers deal with the problems of differential drive mobile robots. The current manuscript emphasizes on how the tracking and stabilization problems can be addressed for a conventional vehicle with Ackermann steering using a state feedback control law. In addition, here we provide the proof of existence of such a control law for a general class of nonlinear affine systems. Note that the proposed control law can be coupled with supervisor controllers, such as the one presented in [40], to tackle vehicle slipage, which is not considered here in order to avoid additional technical challenges.

The rest of the paper is outlined as follows. Section 2 illustrates the kinematic model of a vehicle with Ackermann steering followed by the formulation of stabilization and trajectory tracking problems as a unified manner. The main contribution of this paper, which is the optimal smooth time-varying state feedback law, is described in section 3. A thorough evaluation of the current work with some numerical computer simulations is presented in section 4. Finally, conclusions with some future research avenue are drawn in section 5 .

\section{Vehicle Model and Problem Formulation}

Fig. 1 shows the kinematic model of an Ackermann steering vehicle, where $\left(x_{r}, y_{r}\right)$ and $\left(x_{f}, y_{f}\right)$ are the active points of the rear and front wheels, respectively. Without loss of generality, its configuration at time $t \geq 0$ is represented by the vector $\mathbf{q}(t)=\left[\begin{array}{lll}x(t) & y(t) \theta(t) \phi(t)\end{array}\right]^{T} \in$ $\mathcal{Q} \subset \mathbb{R}^{4}$, where $(x(t), y(t))$ is the Cartesian position of the midpoint of the line of length $2 l$ connecting two axles dividing at their midpoints, $\theta(t) \in(-\pi, \pi]$ is the body orientation 
with respect to $\mathrm{X}$-axis, and $\phi(t) \in(-\pi / 2, \pi / 2)$ is the steering angle of the front wheels with respect to the vehicle body. The vehicle is subject to the nonholonomic constraints given by

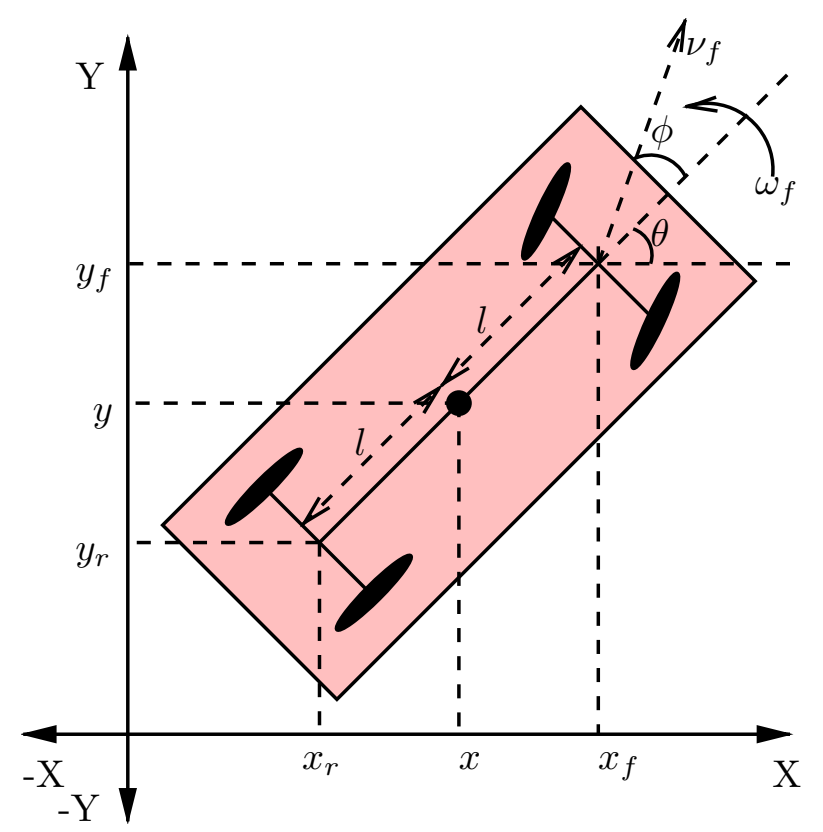

Figure 1: Kinematic model of a vehicle with Ackermann geometry.

$$
\dot{x} \sin (\theta+\phi)-\dot{y} \cos (\theta+\phi)-l \dot{\theta} \cos \phi=0 \text { and } \dot{x} \sin \theta-\dot{y} \cos \theta=0,
$$

which act on each wheel to prevent it from slipping laterally. The constraints (1) can be used to derive the vehicle's (front wheel driving) kinematic model

$$
\dot{\mathbf{q}}(t)=\left[\begin{array}{cc}
\cos \phi(t) \cos \theta(t) & 0 \\
\cos \phi(t) \sin \theta(t) & 0 \\
\frac{1}{l} \sin \phi(t) & 0 \\
0 & 1
\end{array}\right]\left[\begin{array}{l}
\nu_{f}(t) \\
\omega_{f}(t)
\end{array}\right] \equiv \mathbf{F}[\mathbf{q}(t)] \mathbf{u}(t),
$$

where the vehicle's control input vector $\mathbf{u}(t) \equiv\left[\nu_{f}(t) \omega_{f}(t)\right]^{T} \in \mathcal{U} \subset \mathbb{R}^{2}$, with $\nu_{f}(t)$ and $\omega_{f}(t)$ being the front wheels' linear and steering velocities, respectively. In addition, due 
to the vehicle's limit on its velocities, the inputs are constrained as $\left|\nu_{f}(t)\right| \leq \nu_{f}^{\max }$, and $\left|\omega_{f}(t)\right| \leq \omega_{f}^{\max }$ for $t \in I \equiv\left[0, t_{f}\right], t_{f}>0$, where $\nu_{f}^{\max }$ and $\omega_{f}^{\max }$ is the maximum linear and steering velocities of the vehicle. Let $\mathbf{q}^{d}(t)$ be the desired (reference) trajectory that the vehicle is supposed to track and $e(t)=\left\|\mathbf{q}(t)-\mathbf{q}^{d}(t)\right\|_{\mathbb{R}^{2}}$ denotes its position tracking error, for $t \in I$. The objective is to find the optimal control input $\mathbf{u}(t) \in \mathcal{U}_{a d} \subset \mathbb{R}^{2}$ that governs the state trajectory $\mathbf{q}(t) \in \mathcal{Q}$ while minimizing the average cumulative position tracking error $\mathscr{E}_{\text {avg }}=\frac{1}{t_{f}} \int_{0}^{t_{f}} e(t) \mathrm{dt}, \quad t_{f}>0$. Given the vehicle's velocity constraint and its nonholonimic constraint (1), the problem can be stated as follows:

$$
\inf _{\left\{\mathbf{q} \in \mathcal{Q}, \mathbf{u} \in \mathcal{U}_{a d}\right\}}\left[\mathscr{E}_{\text {avg }}\right]
$$

\section{Linear Time-Varying State-Feedback Law}

This section illustrates the design procedure of the optimal feedback gain, $\mathbf{K}(t)$, which is the main contribution of this manuscript. For that, the linear state feedback control law is defined as

$$
\mathbf{u}(t)=\mathbf{K}(t) \mathbf{q}(t)
$$

subject to (1), where $\mathbf{K}(t) \neq \mathbf{0}, t \in I$, is the feedback gain for the vehicle model (2). Assuming the fact that the sets $\mathcal{U}$ and $\mathcal{Q}$ are convex, $\mathbf{K}(t)$ must be chosen from a convex set $\mathcal{K} \subset \mathbb{R}^{2 \times 4}$. Furthermore, due to constraint on the vehicle velocities, $\mathbf{K}(t)$ has to be chosen from the admissible matrix space $\mathcal{K}_{a d} \subset \mathcal{K}$. Substituting (4) in (2), yields the following full-state feedback system:

$$
\dot{\mathbf{q}}(t)=\mathbf{F}[\mathbf{q}(t)] \mathbf{K}(t) \mathbf{q}(t) \equiv \mathbf{f}[\mathbf{q}(t), \mathbf{K}(t)], \mathbf{q}(0)=\mathbf{q}_{0},
$$


where $\mathbf{q}_{0} \neq \mathbf{0}$ since the model (5) is a nonlinear homogeneous equation (drift-free system). We emphasize that the linear time-varying gain matrix $\mathbf{K}(t)$ completely characterizes the feedback law (4) for solving the both tracking and stabilization problems. In order to solve both control problems (stabilization and tracking) as a unified manner, let us introduce the cost functional as

$$
\begin{aligned}
& J(\mathbf{K})=\Phi\left[t_{f}, \mathbf{q}\left(t_{f}\right)\right]+\int_{0}^{t_{f}} \ell[t, \mathbf{q}(t, \mathbf{K})] \mathrm{dt}= \\
& \quad \frac{1}{2}\left[\mathbf{q}\left(t_{f}\right)-\mathbf{q}^{d}\left(t_{f}\right)\right]^{T} \mathbf{P}\left(t_{f}\right)\left[\mathbf{q}\left(t_{f}\right)-\mathbf{q}^{d}\left(t_{f}\right)\right]+\frac{1}{2} \int_{0}^{t_{f}}\left[\mathbf{q}(t)-\mathbf{q}^{d}(t)\right]^{T} \mathbf{Q}(t)\left[\mathbf{q}(t)-\mathbf{q}^{d}(t)\right] \mathrm{dt}
\end{aligned}
$$

where the stabilization weight $\mathbf{P}\left(t_{f}\right) \in \mathbb{R}^{4 \times 4}$ and the tracking weight $\mathbf{Q}(t) \in \mathbb{R}^{4 \times 4}$ are symmetric positive definite matrices that indicate the relative importance of the error components along $\mathbb{R}^{4}$. If the vehicle's purpose is to stabilize on a fixed configuration, then $\mathbf{P}\left(t_{f}\right)$ must be higher than $\mathbf{Q}(t)$ and the desired trajectory is set as a fixed configuration. However, the opposite is true for the vehicle to track a desired trajectory. The performance index $J(\mathbf{K})$ in (6) depends on the feedback gain $\mathbf{K}(t)$ through the state variable $\mathbf{q}(t)$ as it is clear from the feedback system (5). Note that $\Phi[\cdot]$ and $\ell[\cdot]$ of (6) are represent the stabilization and tracking cost functionals, respectively. The task now becomes solving the following regulator problem:

$$
\inf _{\mathbf{K} \in \mathcal{K}_{a d}}[J(\mathbf{K})]
$$

which yields $\mathbf{q}(t) \longrightarrow \mathbf{q}^{d}(t)$ as $J(\mathbf{K}) \longrightarrow 0$, for $t \in I$. It is important to point out that, solving the problem (7) will eventually solve the problem (3). Hence, the problem (7) can be solved if there exists an optimal feedback gain $\mathbf{K}^{*}(t)$, for $t \in I$.

Theorem 1 (Existence of optimal feedback gain $\mathbf{K}^{*}(t)$ ). Given the feedback system (5), there exists an optimal feedback gain $\mathbf{K}^{*}(t) \in \mathcal{K}_{\text {ad }}$ that solves the regulator problem (7).

Proof. Using the well known Alaoglu's theorem, $\mathcal{K}_{a d} \subset \mathcal{K} \subset \mathbb{R}^{2 \times 4}$ is a (weak star) $w^{*}$ 
compact set and it suffices to prove that $\mathbf{K} \longmapsto J(\mathbf{K})$ is sequentially weak star continuous. Let $\left\{\mathbf{K}^{i}, i \in \mathcal{N}\right\} \in \mathcal{K}_{a d}$ be a sequence and suppose $\mathbf{K}^{i} \stackrel{w^{*}}{\longmapsto} \mathbf{K}^{*}$. Since $\mathcal{K}_{a d}$ is $w^{*}$ closed, we have $\mathbf{K}^{*} \in \mathcal{K}_{a d}$, see [36]. Suppressing the variable $t$ for clarity, let $\left\{\mathbf{q}^{i}, i \in \mathcal{N}\right\}$ and $\mathbf{q}^{*}$ denote the solutions of the system (5) corresponding to $\left\{\mathbf{K}^{i}, i \in \mathcal{N}\right\}$ and $\mathbf{K}^{*}$, respectively. Hence, the corresponding state equation becomes $\dot{\mathbf{q}}^{i}=\mathbf{f}\left(\mathbf{q}^{i}, \mathbf{K}^{i}\right)$ and $\dot{\mathbf{q}}^{*}=\mathbf{f}\left(\mathbf{q}^{*}, \mathbf{K}^{*}\right)$, with initial conditions $\mathbf{q}^{i}(0)=\mathbf{q}^{*}(0)=\mathbf{q}_{0}$. The solutions of these two state-space models can be described by $\mathbf{q}^{i}(t)=\mathbf{q}_{0}+\int_{0}^{t} \mathbf{f}\left[\mathbf{q}^{i}(\tau), \mathbf{K}^{i}(\tau)\right] d \tau$, and $\mathbf{q}^{*}(t)=\mathbf{q}_{0}+\int_{0}^{t} \mathbf{f}\left[\mathbf{q}^{*}(\tau), \mathbf{K}^{*}(\tau)\right] d \tau$. Subtracting one from another, we get

$$
\mathbf{q}^{i}(t)-\mathbf{q}^{*}(t)=\int_{0}^{t}\left\{\mathbf{f}\left[\mathbf{q}^{i}(\tau), \mathbf{K}^{i}(\tau)\right]-\mathbf{f}\left[\mathbf{q}^{*}(\tau), \mathbf{K}^{*}(\tau)\right]\right\} d \tau
$$

Note that $\mathbf{F}\left(\mathbf{q}^{i}\right), i \in \mathcal{N}$ and $\mathbf{F}\left(\mathbf{q}^{*}\right)$ are uniformly bounded functions and satisfy Lipschitz condition $\left\|\mathbf{F}\left(\mathbf{q}^{i}\right)-\mathbf{F}\left(\mathbf{q}^{*}\right)\right\| \leq L_{c}\left\|\mathbf{q}^{i}-\mathbf{q}^{*}\right\|$, where $L_{c}$ is the Lipschitz constant. Taking the Euclidean norm in both sides of expression (8) and using the triangle inequality yield

$\left\|\mathbf{q}^{i}(t)-\mathbf{q}^{*}(t)\right\| \leq v^{i}(t)+\int_{0}^{t} \beta(\tau)\left\|\mathbf{q}^{i}(\tau)-\mathbf{q}^{*}(\tau)\right\| d \tau$, where $v^{i}(t)=\left\|\int_{0}^{t}\left[\mathbf{F}\left(\mathbf{q}^{i}\right)\left(\mathbf{K}^{i}-\mathbf{K}^{*}\right) \mathbf{q}^{i}\right] d \tau\right\|$,

$\beta(t)=\left(c_{1}(t)+c_{2}(t) L_{c}\right) \in \mathcal{L}_{1}^{+}(I)$, with $\left.c_{1}(t)=\sup \left\{\left\|\mathbf{F}\left(\mathbf{q}^{i}\right)\right\|\right\}\right][\sup \{\|\mathbf{K}\|\}], c_{2}(t)=\sup \{\|\mathbf{K}\|\|\mathbf{q}\|\}$, for $i \in \mathcal{N}, \mathbf{K} \in \mathcal{K}$, and $\mathbf{q} \in \mathcal{Q}$ (see [2, p. 273] for more details). Thus, it follows from Gronwall inequality that $\left\|\mathbf{q}^{i}(t)-\mathbf{q}^{*}(t)\right\| \leq v^{i}(t)+\int_{0}^{t} \exp \left\{\int_{\tau}^{t} \beta\left(\tau_{1}\right) d \tau_{1}\right\} \beta(\tau) v^{i}(\tau) d \tau$. Clearly, $v^{i}(t) \rightarrow 0$, for $t \in I, i \in \mathcal{N}$, as $\mathbf{K}^{i} \stackrel{w^{*}}{\longmapsto} \mathbf{K}^{*}$. Hence, $\mathbf{q}^{i} \stackrel{\mathbf{K}^{*}}{\longmapsto} \mathbf{q}^{*}$. Since both $\ell(t, \cdot)$ and $\Phi(t, \cdot)$ are continuous on $\mathbb{R}^{4}$, we have $\ell\left[t, \mathbf{q}_{i}(t)\right] \longrightarrow \ell\left[t, \mathbf{q}^{*}(t)\right]$ for almost all $t \in I$ and $\Phi\left[t, \mathbf{q}^{i}\left(t_{f}\right)\right] \longrightarrow \Phi\left[t, \mathbf{q}^{*}\left(t_{f}\right)\right]$ as $i \rightarrow \infty$. Thus it follows from the expression (6) that $\lim _{i \rightarrow \infty} J\left(\mathbf{K}^{i}\right)=J\left(\mathbf{K}^{*}\right)$ proving weak star continuity of $J$ on $\mathcal{K}_{a d}$. Since $\mathcal{K}_{a d}$ weak star compact, $J$ attains its minimum on $\mathcal{K}_{a d}$.

Theorem 1 guarantees that there exists an optimal feedback gain $\mathbf{K}^{*}$ for the system (5). 
To solve for the optimal trajectory that minimizes the objective functional (6), we need to derive the necessary conditions of optimality. These necessary conditions are most readily found if the integrand of the cost functional (6) is recast in terms of Hamiltonian $\mathcal{H}$ : $I \times \mathbb{R}^{4} \times \mathbb{R}^{4} \times \mathbb{R}^{2 \times 4} \longrightarrow \mathbb{R}$, which is expressed by

$$
\mathcal{H}[t, \mathbf{q}(t), \boldsymbol{\psi}(t), \mathbf{K}(t)]=\boldsymbol{\psi}^{T}(t) \mathbf{f}[\mathbf{q}(t), \mathbf{K}(t)]+\ell[t, \mathbf{q}(t)]
$$

where $\boldsymbol{\psi}(t) \in \mathbb{R}^{4}, t \in I$, is a vector of Lagrange multipliers whose elements are the costates of the system [2]. We now derive the necessary conditions of optimality feedback model (5).

Theorem 2 (Necessary Conditions of Optimality ). Since the optimal feedback gain $\mathbf{K}^{*}(t), t \in$ I exists, the optimal trajectory $\mathbf{q}^{*}(t), t \in I$ for the feedback model (5) can be obtained if there exists an optimal multiplier $\boldsymbol{\psi}^{*}(t) \in C\left(I, \mathbb{R}^{4}\right)$ such that the triple $\left\{\mathbf{q}^{*}, \boldsymbol{\psi}^{*}, \mathbf{K}^{*}\right\}$ satisfies the following necessary conditions:

$$
\begin{aligned}
& \mathcal{H}\left[t, \mathbf{q}^{*}(t), \boldsymbol{\psi}^{*}(t), \mathbf{K}(t)\right] \geq \mathcal{H}\left[t, \mathbf{q}^{*}(t), \boldsymbol{\psi}^{*}(t), \mathbf{K}^{*}(t)\right], \quad \mathbf{K}(t) \in \mathcal{K}, t \in I, \\
& \dot{\mathbf{q}}^{*}=\frac{\partial \mathcal{H}}{\partial \boldsymbol{\psi}}\left[t, \mathbf{q}^{*}(t), \boldsymbol{\psi}^{*}(t), \mathbf{K}^{*}(t)\right], \mathbf{q}^{*}(0)=\mathbf{q}_{\mathbf{0}}, t \in I, \\
& \dot{\boldsymbol{\psi}}^{*}=-\frac{\partial \mathcal{H}}{\partial \mathbf{q}}\left[t, \mathbf{q}^{*}(t), \boldsymbol{\psi}^{*}(t), \mathbf{K}^{*}(t), \boldsymbol{\psi}^{*}\left(t_{f}\right)=\frac{\partial \Phi}{\partial \mathbf{q}}\left[t_{f}, \mathbf{q}\left(t_{f}\right)\right] .\right.
\end{aligned}
$$

The detailed proof of this Theorem is similar to the necessary conditions for the feedback law of semi-linear dynamic systems given in our previous publication [38] and is omitted here for conciseness purpose. Theorem 2 states that the feedback gain $\mathbf{K}^{*} \in \mathcal{K}_{a d}$ provides the necessary conditions for the vehicle to determine optimal control inputs for its actuator. In order to solve for $\mathbf{K}^{*}$, we express the gradient of the Hamiltonian defined in (9) and set 
it to zero,

$$
\mathcal{H}_{\mathbf{K}} \equiv \frac{\partial \mathcal{H}}{\partial \mathbf{K}}=\mathbf{F}^{T}[\mathbf{q}(t, \mathbf{K})] \boldsymbol{\psi}(t) \mathbf{q}^{T}(t, \mathbf{K})=\mathbf{0}
$$

Note that the expression in $(11)$ is dependent on the gain $\mathbf{K}$ through the solution of the state feedback model (5) for $\mathbf{q}(t, \mathbf{K})$. Hence, the problem boils down to finding $\mathbf{K}(t), t \in I$, such that the vehicle's actual trajectory $\mathbf{q}(t), t \in I$, and the costate trajectory from (10c) satisfy (11). The optimal feedback gain $\mathbf{K}^{*}$ can be determined by satisfying the Hamiltonian inequality 10a). In other words, the choice of $\mathbf{K}$ is to be adaptively tuned to minimize the vehicle's tracking error.

Corollary 1 (Adapting the gain $\mathbf{K}$ ). Consider the vehicle's feedback system (5) defined over the time horizon I. Adapting the gain $\mathbf{K}$ according to the following offline update rule

$$
\mathbf{K}^{\text {new }}=\mathbf{K}^{\text {old }}-\epsilon \mathcal{H}_{\mathbf{K}}, \text { for } 0<\epsilon<1
$$

satisfies the Hamiltonian inequality (10a) and, hence, guarantees the convergence of the vehicle's trajectory to follow its reference trajectory or stabilize on a fixed configuration.

See [38] for its detailed proof. In the following, we numerically solve for the gain $\mathbf{K}$ such that (11) is satisfied, aggregating the components described earlier. Let $\mathbf{K}_{i} \equiv \mathbf{K}_{i}(t), t \in I$, be the gain at the $i$-th iteration of the optimization procedure. Find the optimal gain $\mathbf{K}^{*}$ by repeating Steps $\mathbf{1 - 5}$ until the stopping criterion in Step $\mathbf{5}$ is met.

Step 1: Integrate the vehicle's feedback system (5) with $\mathbf{K} \equiv \mathbf{K}_{\mathbf{i}}(t), t \in I$.

Step 2: Solve costate equation (10c) backward for $\boldsymbol{\psi}_{i}$.

Step 3: Define the Hamiltonian $\mathcal{H}\left(\mathbf{q}_{i}, \boldsymbol{\psi}_{i}, \mathbf{K}_{i}\right)$ as in (9).

Step 4: Compute the cost function $J\left(\mathbf{K}_{i}\right)$ using (6), the gradients of the Hamiltonian $\mathcal{H}_{\mathbf{K}}$ using (11), and its corresponding intergrated norm $\int_{0}^{t_{f}}\left\|\mathcal{H}_{\mathbf{K}}\right\|^{2} \mathrm{dt}$. 
Step 5: If $J\left(\mathbf{K}_{i}\right) \leq \delta_{1}$ or $\int_{0}^{t_{f}}\left\|\mathcal{H}_{\mathbf{K}}\right\|^{2} \mathrm{dt} \leq \delta_{2}$, for pre-defined small positive tolerance constants $\delta_{1}$ and $\delta_{2}$, then $\mathbf{K}_{\mathbf{i}}$ is regarded close enough to its optimal value, and so the algorithm is halted. Otherwise, use the update rule $\mathbf{K}_{i+1}\left(t_{k}\right)=\mathbf{K}_{i}\left(t_{k}\right)-\epsilon \mathcal{H}_{\mathbf{K}\left(t_{k}\right)}+\lambda \Delta \mathbf{K}_{i}\left(t_{k}\right)$ and $\Delta \mathbf{K}_{i}\left(t_{k}\right)=$ $\mathbf{K}_{i}\left(t_{k}\right)-\mathbf{K}_{i-1}\left(t_{k}\right)$ to adjust the piecewise-constant feedback gain for $t \in\left[t_{k}, t_{k+1},\right] k=$ $0, \ldots, N-1, N$ is the number of subintervals in $I, \epsilon$ is the step size, and $\lambda$ is the momentum constant (for faster convergence).

\section{Simulation Results}

We now illustrate the performance of the proposed optimal feedback controller using a carlike vehicle with the body length of $l=30 \mathrm{~cm}$. The vehicle's velocities are constrained as $|\nu|_{f} \leq \nu_{f}^{\max }=1.5 \mathrm{~m} \cdot \mathrm{s}^{-1}$ and $\left|\omega_{f}\right| \leq \omega_{f}^{\max }=1 \mathrm{rad} \cdot \mathrm{s}^{-1}$. The performance metrics adopted in the current work are the vehicle's state tracking error $\mathbf{q}_{e}(t)=\left[x_{e}(t), y_{e}(t), \theta_{e}(t), \phi_{e}(t)\right]^{T}=$ $\mathbf{q}(t)-\mathbf{q}^{d}(t)$ and the average cumulative position error, $\mathscr{E}_{\text {avg }}$, over the time interval of $I \equiv$ $[0,60] \mathrm{s}$, which allow us to make quantitive assessment of the proposed control method. The elements of the feedback gain matrix $\mathbf{K}$ are initially set to $10^{-4}$. The sampling time period is set to $0.6 \mathrm{~s}$. The optimal feedback gain $\mathbf{K}^{*}(t)$ is computed using the optimization procedure described in Section 3. The controller's performance in solving the vehicle's stabilization and tracking problems is demonstrated in the following sections.

\subsection{Parallel Parking}

As stated in the literature, stabilizing a vehicle on a fixed configuration is more difficult than tracking a reference trajectory. In this section, we present the vehicle's parallel parking ability which is actually the stabilization of the vehicle to a fixed configuration.

The stabilization performance of the proposed control scheme is evaluated by choosing the weight matrices as $\mathbf{P}\left(t_{f}\right)=\operatorname{diag}(1,1,1,1)$ and $\mathbf{Q}(t)=\operatorname{diag}(0.02,0.02,0.02), \forall t \in I$. 
Hence, the stabilization at the a fixed configuration is regarded 50 times as importance as guiding the vehicle towards that configuration. The vehicle's goal is to stabilize at the position of $(x, y)=(1,8) \mathrm{m}$ with the orientation of $0^{\circ}$ and the desired orientation of the front wheels is $45^{\circ}$. The initial position and orientation of the vehicle are $(1,0) \mathrm{m}$ and $0^{\circ}$, respectively. The vehicle's parking performance is summarized in Fig. 2. Fig. 2(a) shows the vehicle's ability to stabilize on its target, where the hollow and solid arrows represent the initial and final poses, respectively. The error, distance between the vehicle and its target, shown in Fig. 2(c) represents how fast the vehicle is approaching towards the target with a final error of $\approx 0$, as expected. The optimal feedback gain, $\mathbf{K}^{*}(t), t \in[0,60] \mathrm{s}$, corresponding to the optimal trajectory is shown in Fig. 2(e). Initially, the values of all eight components of the gain matrix $\mathbf{K}^{*}$ are high due to the initial perturbation of the vehicle from the desired target point. As expected, the gain $\mathbf{K}^{*}(t)$ converges to zero as the vehicle reached to target point. It is important to articulate the fact that the values of $\mathbf{K}^{*}(t)$ at time $t \in[0,60] \mathrm{s}$ are admissible in the sense that the left and right wheel velocities in the feedback control $\mathbf{u}^{*}(t)=\mathbf{K}^{*}(t) \mathbf{q}^{*}(t)$ satisfy the velocity constraints of the vehicle.

\subsection{Trajectory Tracking}

Let us consider that the vehicle has to follow a feasible and smooth desired trajectory given in

terms of cartesian positions $\left(x^{d}(t), y^{d}(t)\right)$, for $t \in I \equiv[060] \mathrm{s}$. For that, the vehicle's desired state trajectory must be generated from $\left(x^{d}(t), y^{d}(t)\right)$. The desired Cartesian trajectory $\left(x^{d}(t), y^{d}(t)\right)$ is feasible when it satisfies the vehicle's desired (reference) model from (2), i.e.,

$$
\dot{\mathbf{q}}^{d}(t)=\mathbf{f}\left[\mathbf{q}^{d}(t), \mathbf{u}^{d}(t)\right],
$$

where $\mathbf{q}^{d}(t)=\left[x^{d}(t) y^{d}(t) \theta^{d}(t) \phi^{d}(t)\right]^{T}$ is the desired state of the vehicle with the suitable initial condition $\mathbf{x}^{d}(0)=\left[x^{d}(0) y^{d}(0) \theta^{d}(0) \phi^{d}(0)\right]^{T}$. We solve for the vehicle's linear veloc- 


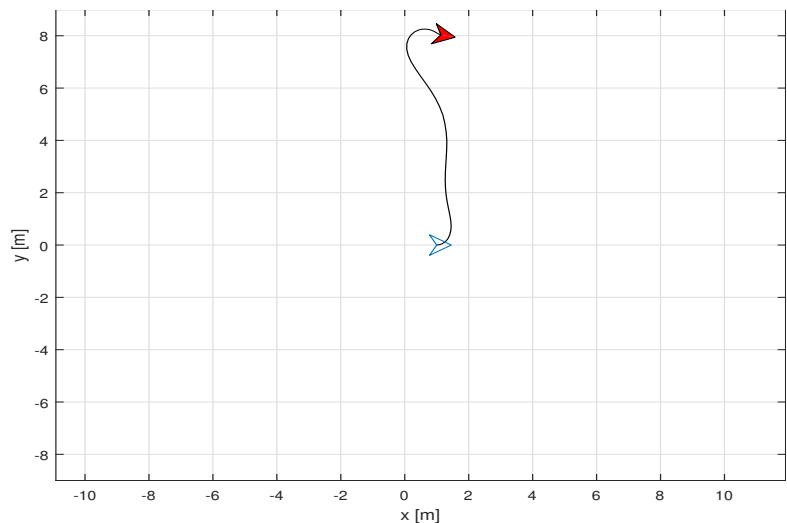

(a)

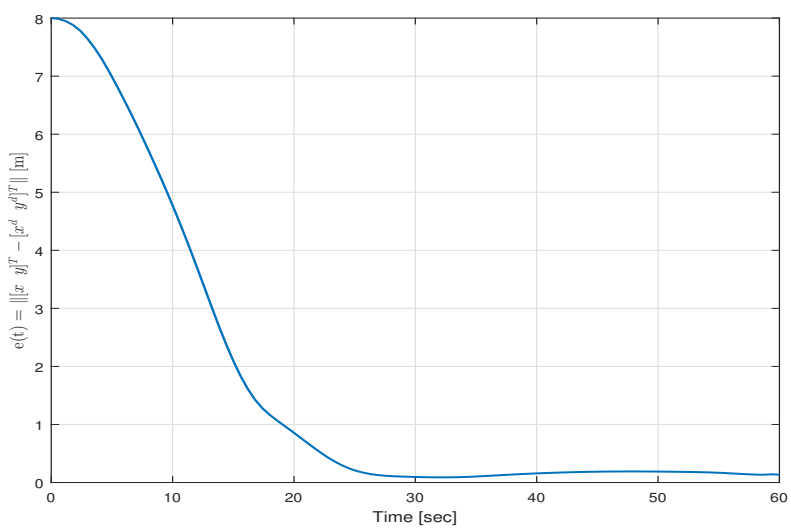

(c)

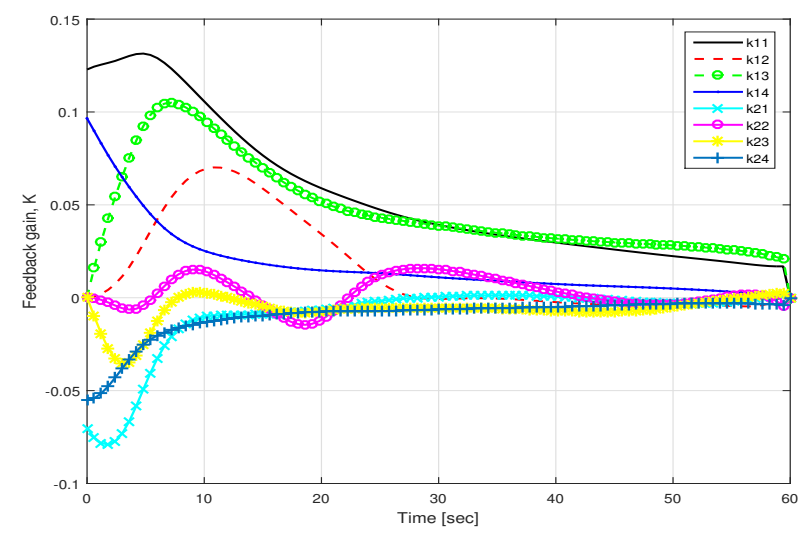

(e)

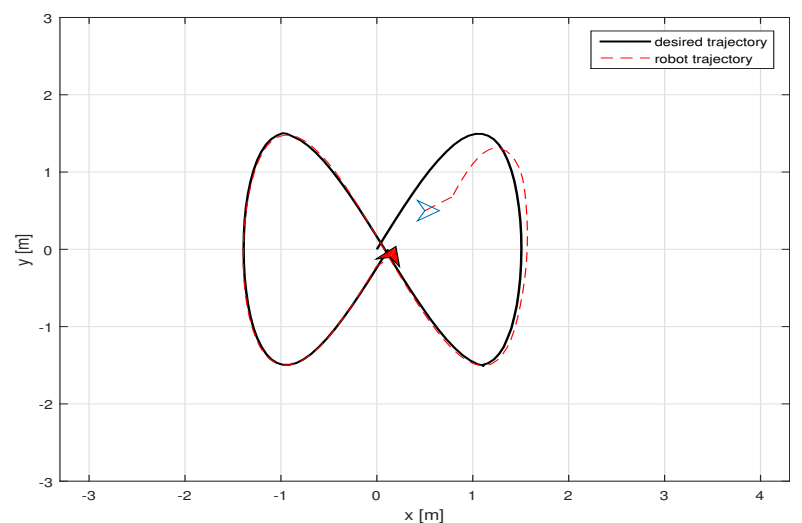

(b)

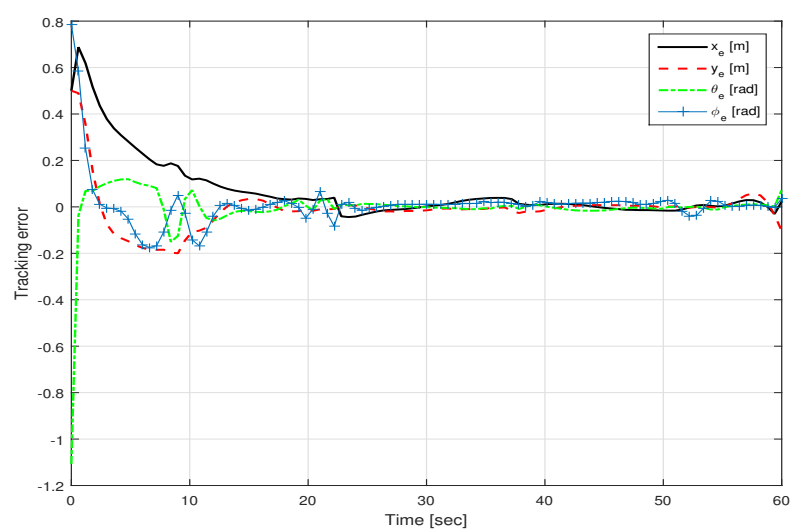

(d)

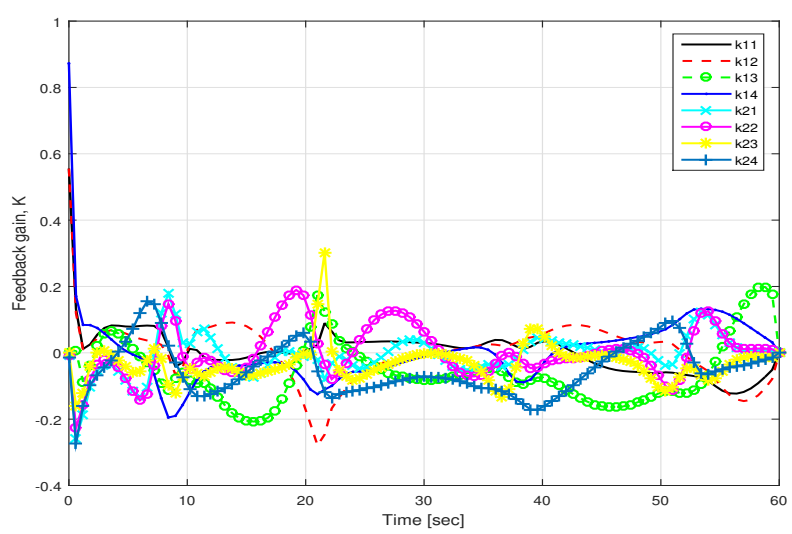

(f)

Figure 2: Controller's performance for parallel parking and trajectory (eight-shaped) tracking problems: (a) \& (b) vehicle's trajectory (hollow arrow: initial state, solid arrow: final state), (c) \& (d) error, and (e) \& (f) optimal time-varying feedback gain, $\mathbf{K}^{*}(t)$. 
ity $\nu^{d}(t)$ (not the front wheel velocity, $\nu_{f}^{d}(t)$ ) and steering velocity $\omega_{f}(t)$ by following the procedure illustrated in [41]. Note that the vehicle's body angle $\theta^{d}(t) \in(-\pi, \pi]$ and its front wheels' orientation $\phi^{d}(t) \in(-\pi / 2, \pi / 2)$. Substituting $\nu^{d}(t)=\nu_{f}^{d}(t) \cos \phi(t)$ and using $\omega_{f}^{d}(t)$ yield the desired state trajectory of the vehicle, which is the solution of the desired model (13). For the vehicle to track the desired state trajectory, the weight matrices of the cost function (6) are chosen as $\mathbf{P}\left(t_{f}\right)=\operatorname{diag}(1,1,2,2)$ and $\mathbf{Q}(t)=\operatorname{diag}(1,1,2,2), \forall t \in I$. Hence, trajectory tracking is given equal importance as just reaching the final destination.

We choose $x^{d}(t)=1.5 \sin (\pi t / 30), y^{d}(t)=1.5 \sin (\pi t / 15)$, for $t \in\left[\begin{array}{ll}060\end{array}\right] \mathrm{s}$, as the reference eight-shaped trajectory and the vehicle's initial state is $\left(0.5,0.5,0,45^{\circ}\right)$. The tracking performance is revealed in Fig. 2, Fig. 2(b) reveals the vehicle's tracking capability in such a complex trajectory, where tracking error (see Fig. 2(d) still remain approximately zero until end of the trajectory. The bounded control velocities are generated from the optimal feedback gain revealed in Fig. 2(f),

Given the satisfactory numerical results for solving tracking and stabilization problems of vehicles with Ackermann steering, the proposed feedback law can be qualitatively compared with the model predictive control law presented in [27] in that the proposed feedback law does not rely on the complexity of the reference trajectory of the vehicle as opposed to [27]. In most cases, see [26, 27], for example, the reference trajectory has to be satisfied by the robot's kinematic model which is not the case considered in the present work.

\section{Conclusion}

In this paper, a novel linear time-varying optimal state feedback control law for solving two main control problems (stabilization and tracking) of a nonholonomic vehicle with Ackermann geometry is proposed. The proposed technique relies on optimizing the linear feedback gain taking into account the vehicle's actuator constraints. The stabilization and tracking problems are successfully solved with sufficiently small error, as expected. It is interesting 
to note that the vehicle model is not required to be linearized to follow a certain reference trajectory in finite time. It is worth pointing out that the theoretical contribution for the proposed control law presented herein opens the door for solving these problems of a general class of nonlinear affine systems.

\section{References}

[1] R. W. Brockett, "Asymptotic stability and feedback stabilization," Differential Geometric Control Theory, pp. 181-191, 1983.

[2] N. U. Ahmed, Dynamic Systems and Control with Applications. New Jersey: World Scientific, 2006.

[3] L. Ssebazza and Y.-J. Pan, "Dgps-based localization and path following approach for outdoor wheeled mobile robots," International Journal of Robotics and Automation, vol. 30, no. 1, 2015.

[4] J. Kim and W. Chung, "Efficient placement of beacons for localization of mobile robots considering the positional uncertainty distributions," International Journal of Robotics and Automation, vol. 30, no. 2, pp. 119-127, 2015.

[5] H. Yamaguchi, "A distributed smooth time-varying feedback control law for multiple nonholonomic mobile robots to make group formations," Transactions of the Society of Instrument and Control Engineers, vol. 39, no. 12, pp. 1108-1116, 2003.

[6] F. Rosales-Hernandez, M. Velasco-Villa, B. d. M.-C. Rafael Castro-Linares, and M. A. Hernndez-Perez, "Synchronization strategy for differentially driven mobile robots: Discrete-time approach," International Journal of Robotics and Automation, vol. 30, no. 1, pp. 50-59, 2015. 
[7] A. Ailon and I. Zohar, "Control strategies for driving a group of nonholonomic kinematic mobile robots in formation along a time-parameterized path," IEEE/ASME Transactions on Mechatronics, vol. 17, no. 2, pp. 326-336, April 2012.

[8] Q. Cui, X. Li, X. Wang, and M. Zhang, "Backstepping control design on the dynamics of the omni-directional mobile robot," Applied Mechanics and Materials, vol. 203, pp. $51-6,2012$.

[9] D. Chwa, "Tracking control of differential-drive wheeled mobile robots using a backstepping-like feedback linearization," IEEE Transactions on Systems, Man and Cybernetics, Part A: Systems and Humans, vol. 40, no. 6, pp. 1285-1295, Nov. 2010.

[10] S. Park and S. Hashimoto, "Autonomous mobile robot navigation using passive RFID in indoor environment," IEEE Transactions on Industrial Electronics, vol. 56, no. 7, pp. 2366-2373, 2009.

[11] F.-L. Lian, Y.-C. Lin, C.-T. Kuo, and J.-H. Jean, "Rate and quality control with embedded coding for mobile robot with visual patrol," IEEE Systems Journal, vol. 6, no. 3, pp. 368-377, Sep. 2012.

[12] D. Chwa, "Sliding-mode tracking control of nonholonomic wheeled mobile robots in polar coordinates," IEEE Transactions on Control Systems Technology, vol. 12, no. 4, pp. 637-644, Jul. 2004.

[13] C.-L. Hwang and N.-W. Chang, "Fuzzy decentralized sliding-mode control of a carlike mobile robot in distributed sensor-network spaces," IEEE Transactions on Fuzzy Systems, vol. 16, no. 1, pp. 97-109, 2008.

[14] M. Rubagotti, M. Della Vedova, and A. Ferrara, "Time-optimal sliding-mode control of a mobile robot in a dynamic environment," IET Control Theory 83 Applications, vol. 5, no. 16, pp. 1916-24, 2011. 
[15] E. J. Rodriguez-Seda, C. Tang, M. W. Spong, and D. M. Stipanovic, "Trajectory tracking with collision avoidance for nonholonomic vehicles with acceleration constraints and limited sensing," International Journal of Control, pp. 1-13, aug 2014. [Online]. Available: http://ijr.sagepub.com/content/early/2014/08/24/0278364914537130

[16] Z. Huaguang, "A novel infinite-time optimal tracking control scheme for a class of discrete-time nonlinear systems via the greedy hdp iteration algorithm," IEEE Transactions on System, Man, and Cybernetics, Part B-Cybernetics, vol. 38, no. 4, pp. 937-942, 2008.

[17] Z. Huaguang, C. Lili, Z. Xin, and L. Yanhong, "Data-driven robust approximate optimal tracking control for unknown general nonlinear systems using adaptive dynamic programming method," IEEE Transactions on Neural Networks, vol. 22, no. 12, pp. 2226-2236, Dec 2011.

[18] Z. Huaguang, C. Lili, and L. Yanhong, "Near-optimal control for nonzero-sum differential games of continuous-time nonlinear systems using single-network adp," IEEE Transactions on Cybernetics, vol. 43, no. 1, pp. 206-216, Feb 2013.

[19] F. ur Rehman, "Steering control of nonholonomic systems with drift: The extended nonholonomic double integrator example," Nonlinear Analysis: Theory, Methods 86 Applications, vol. 62, no. 8, pp. 1498-1515, 2005, hybrid Systems and Applications.

[20] P. Setlur, J. Wagner, D. Dawson, and D. Braganza, "A trajectory tracking steer-bywire control system for ground vehicles," IEEE Transactions on Vehicular Technology, vol. 55, no. 1, pp. 76-85, 2006.

[21] H. Chen, M.-M. Ma, H. Wang, Z.-Y. Liu, and Z.-X. Cai, "Moving horizon $\mathcal{H}_{\infty}$ tracking control of wheeled mobile robots with actuator saturation," IEEE Transactions on Control Systems Technology, vol. 17, no. 2, pp. 449-57, Mar. 2009. 
[22] D. Gu and H. Hu, "Receding horizon tracking control of wheeled mobile robots," IEEE Transactions on Control Systems Technology, vol. 14, no. 4, pp. 743-749, july 2006.

[23] K. B. Kim and B. K. Kim, "Minimum-time trajectory for three-wheeled omnidirectional mobile robots following a bounded-curvature path with a referenced heading profile," IEEE Transactions on Robotics, vol. 27, no. 4, pp. 800-808, aug. 2011.

[24] R. Gonzalez, M. Fiacchini, T. Alamo, J. Guzman, and F. Rodriguez, "Online robust tube-based mpc for time-varying systems: a practical approach," International Journal of Control, vol. 84, no. 6, pp. 1157-70, 2011.

[25] Z. Li, H. Xiao, C. Yang, and Y. Zhao, "Model predictive control of nonholonomic chained systems using general projection neural networks optimization," IEEE Transactions on Systems, Man, and Cybernetics: Systems, vol. 45, no. 10, pp. 1313-1321, Oct 2015.

[26] Z. Li, C. Yang, C.-Y. Su, J. Deng, and W. Zhang, "Vision-based model predictive control for steering of a nonholonomic mobile robot," IEEE Transactions on Control Systems Technology, vol. -, no. 99, pp. 1-, 2015, accepted for publication.

[27] Z. Li, J. Deng, R. Lu, Y. Xu, J. Bai, and C.-Y. Su, "Trajectory-tracking control of mobile robot systems incorporating neural-dynamic optimized model predictive approach," IEEE Transactions on Systems, Man, and Cybernetics: Systems, vol. -, no. 99, pp. 1-, 2015, accepted for publication.

[28] K.-C. Cao and Y.-P. Tian, "A time-varying cascaded design for trajectory tracking control of non-holonomic systems," International Journal of Control, vol. 80, no. 3, pp. 416-29, 2007.

[29] A. P. Aguiar and J. P. Hespanha, "Trajectory-tracking and path-following of underactuated autonomous vehicles with parametric modeling uncertainty," IEEE Transactions on Automatic Control, vol. 52, no. 8, pp. 1362-1379, Aug. 2007. 
[30] B. S. Park, S. J. Yoo, J. B. Park, and Y. H. Choi, "A simple adaptive control approach for trajectory tracking of electrically driven nonholonomic mobile robots," IEEE Transactions on Control Systems Technology, vol. 18, no. 5, pp. 1199 -1206, sept. 2010.

[31] M. H. Amoozgar, S. H. Sadati, and K. Alipour, "Trajectory tracking of wheeled mobile robots using a kinematical fuzzy controller," International Journal of Control, vol. 27, no. 1 , pp. 49-59, 2012.

[32] M. Aicardi, G. Casalino, A. Bicchi, and A. Balestrino, "Closed loop steering of unicycle like vehicles via lyapunov techniques," IEEE Robotics Automation Magazine, vol. 2, no. 1, pp. 27-35, mar 1995.

[33] J.-B. Pomet, "Explicit design of time-varying stabilizing control laws for a class of controllable systems without drift," Systems \&3 Control Letters, vol. 18, no. 2, pp. 147158, Feb. 1992.

[34] Z. Cao, Y. Zhao, and S. Wang, "Trajectory tracking and point stabilization of noholonomic mobile robot," in IEEE/RSJ International Conference on Intelligent Robots and Systems, oct. 2010, pp. $1328-1333$.

[35] P. Morin and C. Samson, "Control of nonholonomic mobile robots based on the transverse function approach," IEEE Transactions on Robotics, vol. 25, no. 5, pp. 1058-73, Oct. 2009.

[36] N. U. Ahmed and M. S. Miah, "Optimal feedback control law for a class of partially observed uncertain dynamic systems: A min-max problem," Dynamic Systems and Applications, vol. 20, no. 1, pp. 149-167, 2011.

[37] M. S. Miah and W. Gueaieb, "Optimal time-varying p-controller for a class of uncertain nonlinear systems," International Journal of Control, Automation and Systems, vol. 12, no. 4, August 2014. 
[38] — - "Mobile robot trajectory tracking using noisy rss measurements: An rfid approach," ISA Transactions: The Journal of Automation, Elsevier, vol. 53, no. 2, pp. 433-443, March 2014.

[39] — _ "Rfid-based mobile robot trajectory tracking and point stabilization through online neighboring optimal control," Journal of Intelligent and Robotic Systems, vol. 78, no. 3-4, pp. 377-399, June 2015.

[40] J. Broderick, D. Tilbury, and E. Atkins, "Supervisory traction control for a slipping ugv," in American Control Conference (ACC), 2013, June 2013, pp. 4350-4355.

[41] A. D. Luca, G. Oriolo, and C. Samson, "Feedback control of a nonholonomic carlike robot," in Robot Motion Planning and Control, ser. Lecture Notes in Control and Information Sciences, J.-P. Laumond, Ed. Springer, 2000, vol. 229, ch. 4, pp. 170-253. 